\title{
A NOTE ON THE GEOMETRIC CRITERIA FOR THE FACTORIALITY OF AN AFFINE RING
}

\author{
JAMES HORNELL
}

\begin{abstract}
Let $R$ be a normal integral domain finitely generated over a field $k$, let $U$ be an affine algebraic variety with coordinate ring $R$, and let $V$ be a projective completion of $U$ over $k . R$ is factorial if and only if the class group of $V$ over $k$ is generated by the $k$-rational cycles at infinity with respect to $U$. If $k$ is algebraically closed, $R$ is shown to be factorial if and only if the Picard group of $V$ is zero and the Néron-Severi group of $V$ is generated by the $k$-rational cycles at infinity. If $k$ is finitely generated over its prime field, some well-known arithmetic results are applied to show the existence of affine localizations of $V$ which have a factorial coordinate ring over $k$. The relationship between the existence of an affine localization of $V$ with a factorial coordinate ring, and the birationality of $V$ is also discussed.
\end{abstract}

One would like to have geometric criteria for the coordinate ring of an irreducible algebraic variety defined over a field $k$, a finitely generated integral domain over $k$, to be factorial. I am indebted to Professor A. Seidenberg for bringing this problem to my attention. For the case of a curve the theorems below are similar to Cunnea's Theorems 4.1 and 4.5 of [4], but the proof there given using the Riemann-Roch theorem is not applicable to higher dimension.

1. The geometric criteria. Let $U$ be a closed subvariety of an affine $n$ space $A^{n}$ contained in a projective $n$-space $P^{n}$ with $L$ being the linear form vanishing on the hyperplane at infinity with respect to $A^{n}$. The class group of the closure $V$ of $U$ in $P^{n}$ will play the essential role. The following is a slightly more general formulation.

Let $V$ be a projective algebraic variety, defined and irreducible over the field $k, k[V]$ its homogeneous coordinate ring over $k$, and $k(V)$ its function field over $k$. Let $D(V)$ be the group of divisors on $V$ over $k$, the free abelian group generated by the $k$-valuations of $k(V)$ which have center of codimension one on $V$, here called the prime divisors of $V$ over $k$. If $f \in k(V)$ is nonzero let $(f)=\Sigma_{v} v(f) v$, where the sum is over all prime divisors $v$ of $V$ over $k$. These divisors of form $(f)$ are called the principal divisors of $V$ over $k$, and they form the subgroup $D_{l}(V)$ of $D(V)$ called the subgroup of divisors linearly equivalent to zero over $k_{\text {. Let }} D_{a}(V)$ be the subgroup of divisors

Received by the editors May 30, 1973 and, in revised form, September 18, 1974. AMS (MOS) subject classifications (1970). Primary 13F15; Secondary 14C20. 
algebraically equivalent to zero on $V$ or equivalently on the $k$-normalization of $V$. (For the usual form of the definition of algebraic equivalence see [7, Chapter III, §1].)

There is another, different definition of the group of divisors of $V$ over $k$, the free abelian group generated by the $k$-irreducible codimension one $k$ subvarieties of $V$ (the homogeneous height one prime ideals of $k[V]$ ). In general, this group is a subgroup of the former group, for identify each $k$ irreducible codimension one $k$-subvariety $W$ of $V$ with $\Sigma_{v}\left[k_{v}: k_{W}\right] v$, where the sum is over all $k$-valuations $v$ of $k(V)$ centered at $W$, where $k_{v}$ is the residue field of the valuation ring of $v$, and where $k_{W}$ is the residue field of the local ring of $V$ at $W$ over $k$. (Compare this to the projection formula [21, Theorem 10, p. 203], [5] and [16, Chapter V, \$C] applied to the $k$ normalization of $V$.) If $V$ is $k$-normal the two groups are equal.

Let $F$ be a nonconstant homogeneous element of $k[V]$, and let $V(F)$ be the locus of $F$ on $V . V_{F}=V \sim V(F)$ is affine with coordinate ring $k\left[V_{F}\right]=$ $\left\{G / F^{s} \mid s>0\right.$ and $G \in k[V]$ homogeneous with $\left.\operatorname{deg} G=s \operatorname{deg} F\right\}$. Assume that $V_{F}$ is normal over $k$. Then $V_{F}$ is also an affine open subset of the $k$ normalization of $V$. If $A \in D(V)$ let $A_{F}$ be the sum of the components of $A$ which have support not contained in $V(F)$. If $S \subset D(V)$ let $S_{F}=\left\{A_{F} \mid A \in S\right\}$. The divisor group of $k\left[V_{F}\right], D\left(V_{F}\right)$, is $D(V)_{F}$, and let $P\left(V_{F}\right)$ be the subgroup of principal divisors of $k\left[V_{F}\right] . \quad P\left(V_{F}\right)=D_{l}(V)_{F} \subset D_{a}(V)_{F}$. The following diagram is commutative and exact

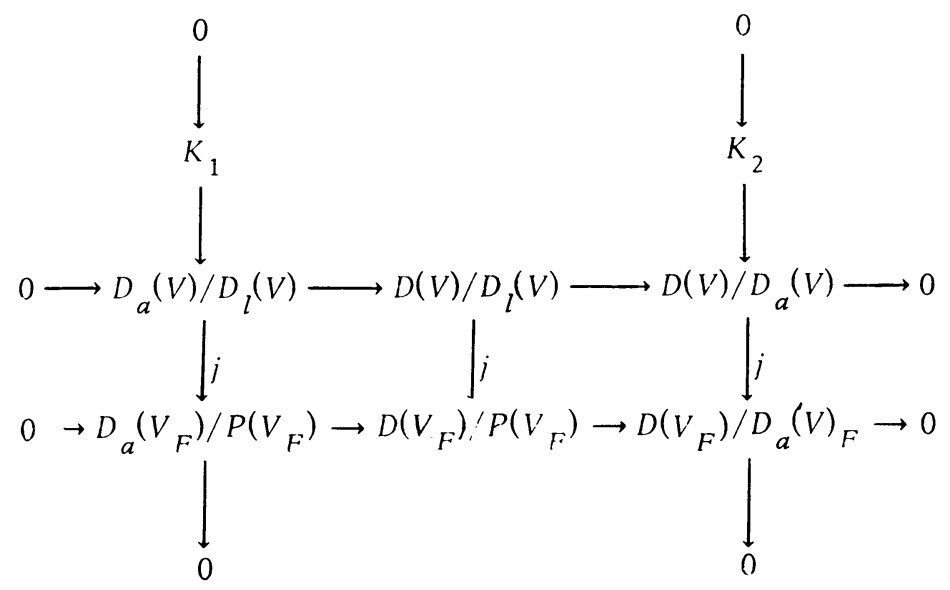

where $j(A)=A_{F}$ for all $A \in D(V)$. The kernel $K_{1}$ is the subset of elements of $D_{a}(V)$ which are linearly equivalent to a divisor with support contained in $V(F)$, and $K_{1}$ is thus finitely generated. The kernel $K_{2}$ is generated by the elements of $D(V)$ which have support contained in $V(F)$.

Recall the fact that a noetherian integral domain $R$ is factorial if and only if each of its prime ideals of height one is principal, which in turn 
holds if and only if $R$ is integrally closed in its quotient field and has zero class group. (The first equivalence is easily proven using the principal ideal theorem [22, Theorem 29, p. 238], and the second equivalence follows from the first by using the normality of $R$.).

The class group of $k\left[V_{F}\right]$ is $D\left(V_{F}\right) / P\left(V_{F}\right)$, and Theorem 1 below now follows from the remarks made on the above diagram.

Theorem 1. Let $V$ be a projective algebraic variety, defined and irreducible over $k$. Let $F \in k[V]$ be homogeneous and nonconstant, and assume that $V_{F}$ is normal over $k$. Then $k\left[V_{F}\right]$ is factorial if and only if $D_{a}(V) / D_{l}(V)$ is generated by divisors algebraically equivalent to zero with support contained in $V(F)$ and $D(V) / D_{a}(V)$ is generated by divisors with support contained in $V(F)$.

2. The application of some well-known arithmetic results. For Theorem 1 and the preceding discussion the base field $k$ had been fixed. Now let $V$ be, in addition, irreducible, although zero divisors may be treated as in [6], and let $K$ be an extension field of $k$. Identify each $k$-valuation $v$ of $k(V)$ with $\Sigma_{w}\left(\left[k_{v}: k\right]_{\iota} /\left[k_{w}: k\right]_{\imath}\right) w$, where the sum is over the distinct $K$-extensions $w$ of $v$ to $K(V)$, and where $\left[k_{v}: k\right]_{\iota}$ is the order of inseparability of $k_{v}$ over $k$ [19 or 21, p. 21$]$.

Let $D^{k}(V)$ denote the group of divisors on $V$ over $k$, which was previously denoted by $D(V)$. By the above identification $D^{k}(V) \subset D^{K}(V)$, and the elements of $D^{k}(V)$ are called $k$-divisors or divisors rational over $k$ [19, p. 198], or [22, p. 207]. Let $D_{l}^{k}(V)$ denote the divisors linearly equivalent to zero on $V$ over $k$, previously denoted by $D_{l}(V)$. By [21, Corollary 2, p. 265] $D_{l}^{k}(V)=D_{l}^{K}(V) \cap D^{k}(V)$. Also let $D_{a}^{k}(V)$ denote the $k$-divisors algebraically equivalent to zero, denoted previously by $D_{a}(V)$. Although it was not noted before, $D_{a}^{k}(V)$ is the subgroup of $k$-divisors algebraically equivalent to zero over the algebraic closure of $k$, and thus $D_{a}^{k}(V)=D_{a}^{K}(V) \cap D^{k}(V)$. (See [12, p. 139].)

The Néron-Severi group of $V$ (over $k$ ) is $D^{k}(V) / D_{a}^{k}(V)[7, \mathrm{p} .64]$, and it is finitely generated as an abelian group [8, Theorem $7, \mathrm{p} .86]$, or [11, Theorem 2, p. 145].

$D_{a}^{k}(V) / D_{l}^{k}(V)$ is a subgroup of the abelian variety $\operatorname{Pic}(V)$, the Picard variety of a projective normalization of $V[7, \mathrm{p} .64]$, which we shall also call the Picard variety of $V . \operatorname{Pic}(V)$ is a birational invariant of $V[7, \mathrm{p}$. 152], [10, Theorem 4, p. 231], or [13, Proposition 1, p. 4]. There is a finite extension $k^{\prime}$ of $k$ such that $D_{a}^{k^{\prime}}(V) / D_{l}^{k^{\prime}}(V)$ is the subgroup of $k^{\prime}$-rational points of $\operatorname{Pic}(V)$ [8, p. 86], [13, p. 2].

Let $k$ be a finitely generated extension of its prime field. Then 
$D_{a}^{k}(V) / D_{l}^{k}(V)$ is finitely generated [8, Theorem $1, \mathrm{p} .71$, or Corollary, p. 86]; see also [11, Theorem 3, p. 146] and [18, p. 302]. Let $F$ be a homogeneous element of $k[V]$ which vanishes on the nonnormal locus of $V$ and on the support of each element of a finite set of generators of $D_{a}^{k}(V) / D_{l}^{k}(V)$ and of $D^{k}(V) / D_{a}^{k}(V)$. By Theorem $1, V_{F}=V \sim V(F)$ has a factorial coordinate ring over $k$.

Let us digress and prove the known fact that if $k$ is an algebraically closed field and if $A$ is an abelian variety defined over $k$, then the subgroup of $k$-rational points of $A$ is not a finitely generated group. Take $A$ to be imbedded in a projective space $P^{n}$, and let $A^{n}$ be an affine open of $P^{n}$ with coordinates $X_{1}, \ldots, X_{n}$. It may be assumed that $A \cap A^{n}$ is nonempty. The set $U$ of all $a \in k$ such that $V\left(X_{1}-a\right)$ and $A$ have nonempty intersection in $A^{n}$ is a nonempty Zariski open subset of $k$. (Let $I(A)$ be the ideal of functions in $k\left[X_{1}, \ldots, X_{n}\right]$ which vanish on $A$. Then $A \cap V\left(X_{1}-a\right)$ is empty if and only if $\left(X_{1}-a-1\right) \in I(A), I(A)$ is a proper ideal, so only one such $a$ may exist.) Let $k_{0} \subset k$ be a field of definition of $A$ which is finitely gene-

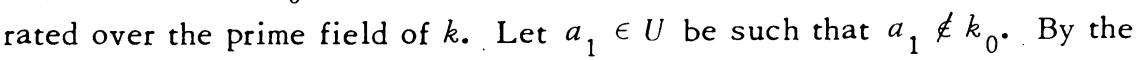
Hilbert Nullstellensatz there is a point $\left(a_{1}, a_{2}, \ldots, a_{n}\right)$ of $A$ which is rational over $k_{\text {. Let }} k_{1}=k_{0}\left(a_{1}, \ldots, a_{n}\right)$. Let $A_{0}$ be the subgroup of $k_{0}-$ rational points of $A$, and let $A_{1}$ be the subgroup of $k_{1}$-rational points of $A$. $A_{0}$ is a proper subgroup of $A_{1} \cdot$. By induction there is an infinite ascending sequence of subfields $k_{0} \subset k_{1} \subset \cdots \subset k_{i-1} \subset k_{i} \subset \cdots$ of $k$, such that, letting $A_{i}$ be the subgroup of $k_{i}$-rational points of $A, A_{0} \subset A_{1} \subset \cdots \subset A_{i-1} \subset$ $A_{i} \subset \cdots$ is an infinite, strictly ascending chain.

Now let the field $k$ contain an algebraically closed subfield, and let $V$ have an affine open subset the coordinate ring of which is factorial over $k$. Then $D_{a}^{k}(V) / D_{l}^{k}(V)$ is either zero or not finitely generated, by Theorem 1 , $D_{a}^{k}(V) / D_{l}^{k}(V)$ is finitely generated, so it must be zero. $D_{a}^{k}(V) / D_{l}^{k}(V)$ is dense in $\operatorname{Pic}(V)$, and $\operatorname{Pic}(V)$ is zero also.

These comments are summarized in Theorem 2 below.

Theorem 2. Let $V$ be an irreducible projective algebraic variety defined over $k$.

If $k$ is finitely generated over its prime field, then $V$ contains an affine open subset which has a factorial coordinate ring over $k$.

If $k$ contains an algebraically closed subfield and if $V$ contains an affine open subset which has a factorial coordinate ring over $k$, then the Picard variety, $\mathrm{Pic}(V)$, of $V$ is zero.

Using the birational invariance of $\operatorname{Pic}(V)$, the projectivity of $V$ may be removed from the hypotheses. The dimension of $\mathrm{Pic}(V)$ has been called the irregularity of $V$. 
Theorem 2 easily gives an example of an affine ring which is factorial and which has an entire, but not factorial, extension to the algebraic closure of the base field (e.g. an appropriate affine open subset of a curve of genus one over the rational field).

3. Rationality: remarks and examples. Let $V$ be projective and birationally equivalent to an $n$-dimensional projective space $P^{n}$ for some $n$. Then $\operatorname{Pic}(V)$ is zero. $\left(\operatorname{Pic}\left(P^{1}\right)\right.$ is easily seen to be zero, and $\operatorname{Pic}\left(P^{n}\right) \subset$ $\operatorname{Pic}\left(P^{1}\right)$ by [10, Proposition 4, p. 224, and Proposition 10, p. 233] or [13, $\$ 2$, pp. 2-4].). Let $F \in k[V]$ be homogeneous, zero on the nonnormal locus of $V$, and zero on the support of each element of a finite set of generators of $D^{k}(V) / D_{a}^{k}(V)$. By Theorem 1 the coordinate ring of $V_{F}$ over $k$ is factorial. Thus, if $V$ is birationally equivalent to $P^{n}$ then $V$ has an affine open subset which has a factorial coordinate ring.

If, in addition to the above, we let $V$ be normal and singular at a subvariety $W$ (necessarily of codimension greater than one on $V$ ) and let $F$ be also nonzero on $W, V_{F}$ is affine with a factorial coordinate ring and has a singular subvariety $W$.

For dimension one the converse of the above is valid over an algebraically closed field $k$. Let $k$ be algebraically closed, let $V$ be of dimension one, denote $V$ by $C$, and suppose $C$ has an affine open subset with factorial coordinate ring over $k$. By Theorem 2, $\mathrm{Pic}(C)$, which is the Jacobian of $C$, is zero. Hence the genus of $C$, which is the dimension of $\operatorname{Pic}(C)$ [20, Theorem 18, p. 67, and Theorem 19, p. 70] is zero, and $C$ is thus birationally equivalent to the projective line $P^{1}$ [2, Chapter II, $\$ 2$, p. 23].

A projective variety $V$ over an algebraically closed field $k$ and of dimension greater than one may have an affine open subset with factorial coordinate ring, and yet $V$ may not be birationally equivalent to a projective space $P^{n}$. As an example, a nonsingular cubic threefold in $P^{4}$ is not birationally equivalent to $P^{3}$ [3, Theorem, p. 350], yet its homogeneous coordinate ring is factorial [9, p. 359], [17, p. 691], and any affine open subset has a factorial coordinate ring over $k$.

\section{BIBLIOGRAPHY}

1. A. Andreotti and P. Salmon, Anelli con unica decomponibilità in fattori primi ed un problemma di intersezioni complete, Monatsh. Math. 61 (1957), 97-142. MR $21 \# 3415$.

2. C. Chevalley, Introduction to the theory of algebraic functions of one variable, Math. Surveys, no. 6, Amer. Math. Soc., Providence, R. 1., 1951. : MR 13, 64.

3. C. H. Clemens and P. A. Griffiths, The intermediate Jacobian of the cubic threefold, Ann. of Math. (2) 95 (1972), 281-356. : MR 46 \#1796.

4. W. M. Cunnea, Unique factorization in algebraic function fields, Illinois J. Math. 8 (1964), 425-438. : MR $31 \# 170$.

5. J. Hornell, Intersection theory in an equicharateristic regular local ring and 
the relative intersection theory, Proc. Amer. Math. Soc. 36 (1972), 8-12. MR 46 \#9040.

6. :- Divisorial complete intersections, Pacific J. Math. 44 (1973), 217227. MR $47 \# 6668$.

7. S. Lang, Abelian varieties, Interscience Tracts in Pure and Appl. Math., no. 7, Interscience, New York, 1959. MR 21 \#4959.

8. - Diophantine geometry, Interscience Tracts in Pure and Appl. Math., no. 11, Interscience, New York, and London, 1962. MR $26 \# 119$.

9. S. Lefshetz, On certain numerical invariants of algebraic varieties with application to abelian varieites, Trans. Amer. Math. Soc. 22 (1921), 327-482.

10. T. Matsusaka, On the algebraic construction of the Picard variety, I, Japan. Math. 21 (1951), 217-235. (1952).: MR 15, 983.

11. A. Néron, Problèmes arithmétiques et géométriques rattachés à la notion du rang d'une courbe algébrique dans un corps, Bull. Soc. Math. France 80 (1952), 101-166. MR 15, 151 .

12. - - Arithmétique et classes de diviseurs sur les variétés algébriques, Proc. Internat. Sympos. on Algebraic Number Theory, Tokyo \& Nikko, 1955;

Science Council of Japan, Tokyo, 1956, pp. 139-154. : MR 18, 600.

13. A. Néron and P. Samuel, La variété de Picard d'une variété normale, Ann. Inst. Fourier (Grenoble) 4 (1952), 1-30 (1954). MR 15, 896.

14. P. Samuel, On unique factorization domains, Illinois J. Math. 5 (1961), 117. MR $22 \# 12121$.

15. - Lectures on unique factorization domains, Tata Inst. Fund. Res. Lectures on Math., no. 30, Tata Institute of Fundamental Research, Bombay, 1964. MR 35 \#5428.

16. J.-P. Serre, Algèbre locale multiplicités, Cours au Collège de France, 1957/58, rédigé par Pierre Gabriel, 2ième éd., Lecture Notes in Math., no. 11, Springer-Verlag, Berlin and New York, 1965.

17. F. Severi, Una proprietà delle forme algebriche prive di punti multipli, Atti Accad. Lincei Rend. Cl. Sci. Fis. Mat. Natur. (5) 15 (1906), 691-696.

18. A. Weil, L'arithmétique sur les courbes algébriques, Acta Math. 52 (1929), $281-315$.

19. - Foundations of algebraic geometry, Amer. Math. Soc. Colloq. Publ., vol. 29, Amer. Math. Soc., Providence, R. 1.,. 1946. MR 9, 303.

20. - - Variétés abéliennes et courbes algébriques, Publ. Inst. Math. Univ. Strasbourg, 8, 1946, Actualités Sci. Indust., no. 1064, Hermann, Paris, 1948. MR 10,621 .

21. - Foundations of algebraic geometry, rev. ed., Amer. Math. Soc. Colloq. Publ., vol. 29, Amer. Math. Soc., Providence, R. I., 1962. MR 26 \#2439.

22. O. Zariski and P. Samuel, Commutative algebra. Vol. I, University Ser. ị Higher Math., Van Nostrand, Princeton, N. J., 1958. : MR 19, 833. 